\title{
DEVELOPMENT AND APPLICATION OF BUNCH-BY-BUNCH MEASUREMENT SYSTEM OF HLS *
}

\author{
J.H. Liu", J.H. Wang, Y.J. Pei, K. Zheng, Y.L. Yang, B.G. Sun \\ NSRL, University of Science and Technology of China, Hefei, Anhui 230029 China
}

\begin{abstract}
This paper is intended to present the newly implemented wideband $(100 \mathrm{MHz})$ bunch oscillation measurement system, which is in nature a different method from the narrow-band $(<5 \mathrm{MHz})$ facilities employed before. Basic formalism and implementation details of the system is introduced to illustrate the function of observing coupled bunch instabilities in time and frequency domain. The designed function includes detecting of transverse oscillation, synchrotron phase oscillation, as well as bunch filling pattern. Some diagnostics results of machine instabilities and application of this system are also discussed.
\end{abstract}

\section{INTRODUCTION}

Most synchrotron radiation facilities are designed to operate in high current, multi-bunch mode which results in an inconvenience that the machine performances can be seriously limited by unstable couple-bunch oscillations excited by transients or noise and sustained by long lasting parasitic resonant modes(high order modes) in the vacuum chamber, mainly in the RF cavities. The high current/brilliance strategy moves the technical challenges elsewhere to the vacuum(beampipe, bellow), RF cavity and measures to cure parasitic instabilities(octopole magnets, wideband feedback, etc). Hefei Light Source is a dedicated electron synchrotron radiation facility, operates with high current beam distributed in many bunches, thus undergoing a number of instabilities during operation. There are evidences that the beam life and stability are affected by coupled bunch oscillations. In this paper, we review the bunched frequency signals in time and frequency domain, its relationship to beam instabilities, present the concept and technology of bunch by bunch wideband measurement, and introduce a number of valuable applications.

\section{SIGNAL RECEIVING AND PROCESSING}

Employing Beam Position Monitor mounted on the storage ring beampipe, one can detect beam signals and extract information like bunch position, filled current and synchrotron phase. Suppose an ultra-relativistic beam in a storage ring with revolution frequency $\mathrm{f}_{0}=\omega_{0} / 2 \pi$, consisting of $\mathrm{M}$ equally spaced bunches, it can oscillate coherently in $\mathrm{M}$ modes, depending upon relationship between phase of individual bunch. Taking Betatron/Synchrotron oscillation, orbit distortion as well as dispersion into consideration, assuming bunch developing only dipole moment transversely, one can represent detected signal as[1]

$$
\begin{array}{r}
f(t)=Q_{b}\left(x_{0}+x_{\beta} \cos \varphi_{\beta}(t)+\eta \delta_{\varepsilon} \cos \left(\omega_{s} t+\phi_{\varepsilon 0}\right)\right) \\
\sum_{n=-\infty}^{\infty} \delta\left(t-n T_{0}-\tau_{s} \cos \left(\omega_{s} t+\phi_{0}\right)\right)
\end{array}
$$

where subscript $\beta$ stands for Betatron oscillation and $\mathrm{s}$ for Synchrotron.

Substituting approximately the equation below into $\phi_{\beta}$

$\varphi_{\beta}=\int \omega_{\beta} d t=\omega_{\beta 0} t+\left(\omega_{\xi}-\omega_{\beta 0}\right) \tau_{s} \cos \left(\omega_{s} t+\phi_{0}\right)+\varphi_{0}$

where $\omega_{\xi}=\frac{\xi}{\eta} \omega_{\beta 0}$

one can obtain

$$
\begin{aligned}
F(\omega)= & \omega_{0} x_{0} Q_{b} \sum_{k=-\infty}^{\infty} e^{j k\left(\phi_{0} \frac{\pi}{2}\right)} J_{k}\left(\omega \tau_{s}\right) \sum_{n=-\infty}^{\infty} \delta\left(\omega-n \omega_{0}-k \omega_{s}\right) \\
& +\frac{\omega_{0}}{2} \sum_{k=-\infty}^{\infty} e^{j k\left(\phi_{0} \frac{\pi}{2}\right)}\left[e^{j \varphi_{0}} J_{k}\left(\left(\omega-\omega_{\xi}+\omega_{\beta}\right) \tau_{s}\right) \sum_{n=-\infty}^{\infty} \delta\left(\omega-n \omega_{0}-k \omega_{s}-\omega_{\beta}\right)\right. \\
& \left.+e^{-j \varphi_{0}} J_{k}\left(\left(\omega+\omega_{\xi}-\omega_{\beta}\right) \tau_{s}\right) \sum_{n=-\infty}^{\infty} \delta\left(\omega-n \omega_{0}-k \omega_{s}+\omega_{\beta}\right)\right]
\end{aligned}
$$

The second term of the above equation denotes coupled bunch oscillation. Provided the phase shifts between the Betatron oscillations of two adjacent bunches satisfy the "coherent" relationship denoted below

$\left(\phi_{b+1}-\phi_{b}\right)=\frac{2 n \pi}{M}$

With $M$ equal bunches, $M$ distinct coherent $\mathrm{CB}$ modes may be excited[2]. Here parameter $\mathrm{n}$ is named "mode number". The similar suggestion applies to longitudinal direction. Taking a closer look at the represented spectrum of Eq, we can be convinced that each mode shows up as a pair of lines in the span of $\mathrm{Mf}_{0}$. That means all modes appear once in any frequency span between $\mathrm{pMf}_{0}$ and $(\mathrm{p}+1 / 2) \mathrm{Mf}_{0}$. It is on this basis that we can determine the least bunch by bunch measurement bandwidth of $\mathrm{f}_{\mathrm{RF}} / 2$.

By using a simplified simulation, We can demonstrate the principle of signal measurement. Provided Gaussian bunch with length $\sigma$, from the capacitive BPM pickup we can obtain

$$
v_{p}(t)=i_{p}(t)^{*} \mathrm{~F}^{-1}\left[\frac{\mathrm{Z}_{0}}{1+\mathrm{j} \omega Z_{0} \mathrm{C}}\right] \approx \frac{d}{d t}\left[\frac{d^{2}}{8 a} \frac{Q_{6}}{\sqrt{2 \pi} \sigma} \frac{t}{\left(\frac{\sigma}{C}\right)^{2}} e^{-\frac{t^{2}}{2\left(\frac{\sigma}{C}\right)^{2}}}\right]
$$

$\mathrm{Y} 1(\mathrm{t})=\mathrm{V}_{\mathrm{p}}(\mathrm{t})+\mathrm{V}_{\mathrm{p}}(\mathrm{t}-\mathrm{T})+\mathrm{V}_{\mathrm{p}}(\mathrm{t}-2 \mathrm{~T})$

$\mathrm{T}=1 /(3 * \mathrm{RF})$ 

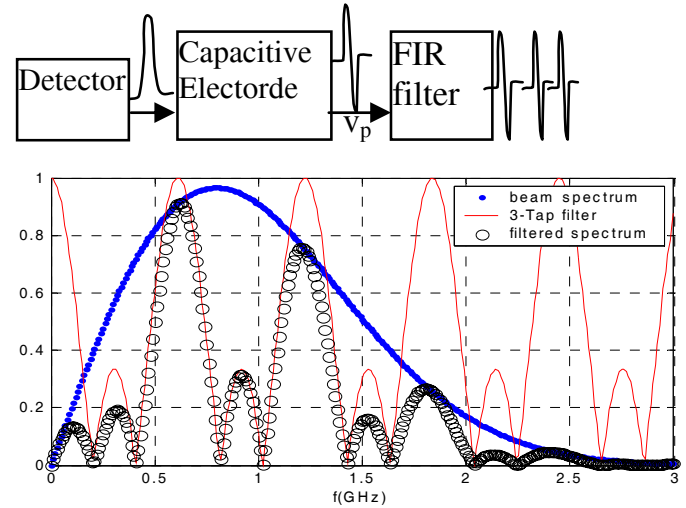

Fig.1 Signal receiving and FIR filtering $\mathrm{Y} 2(\mathrm{t})=\mathrm{Y} 1(\mathrm{t}) \sin \left(\omega_{0} \mathrm{t}\right)=\mathrm{Y} 1(\mathrm{t})\left(\exp \left(\mathrm{j} \omega_{0} \mathrm{t}\right)-\exp \left(-\mathrm{j} \omega_{0} \mathrm{t}\right)\right) / 2 \mathrm{j}$

$\mathrm{Y} 4(\mathrm{t})=\mathrm{Y} 3(\mathrm{t}) * \mathrm{~h}_{100}(\mathrm{t})$

where $h_{100}(t)$ is the impulse response function of $100 \mathrm{MHz}$ Butterworth low pass filter.

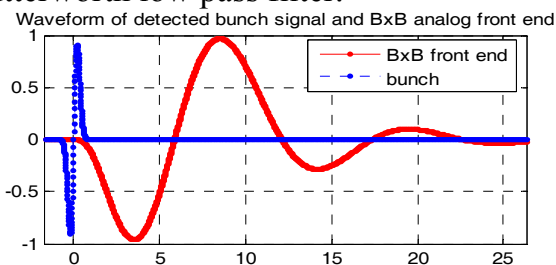

Fig.2 Pickup detected bunch signal and corresponding front-end processing output.

Suppose a beam with $M$ equally spaced bunches, filled charge of individual bunch as resembles the figure below

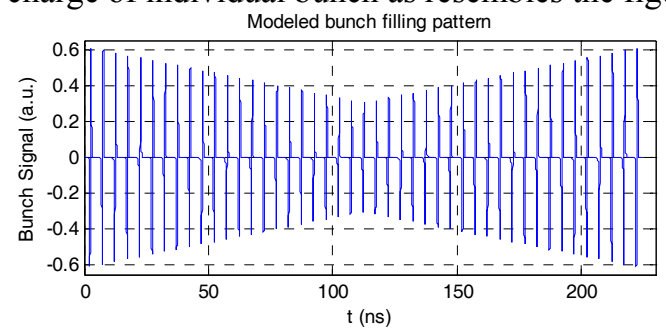

Fig.3 Modeled bunch filling pattern
The obtained bunch by bunch current measurement results becomes as the following figure, in agreement with the suggested filling pattern.

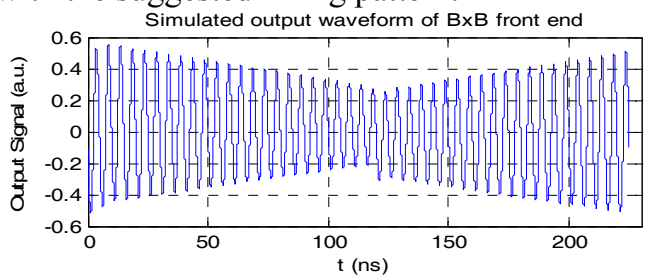

Fig.4 Simulated output waveform of BxB front end.

The similar simulation and processing hold true for the transverse and longitudinal oscillation measurements. On the basis of this knowledge we are convinced that the principle of bunch by bunch measurement is applicable.

\section{BUNCH-BY-BUNCH MEASUREMENT SYSTEM}

Although quite often it is often difficult to find out the exact types and cause of beam instabilities, this widebandwidth bunch oscillation observor acts as eyes of research and bridges the gap by measuring and processing beam signals and converting these scientific or technical subjects to clear and accessible mathematical exposition and illustration. Different from the narrow bandwidth measurement such as COD and Turn-by-Turn system, with bandwidth of $20 \mathrm{KHz}$ and $5 \mathrm{MHz}$ respectively, bunch by bunch measurement is in nature a wide bandwidth one[3]. The circuitry bandwidth is $f_{\mathrm{RF}} / 2$ and data acquisition rate is $\mathrm{f}_{\mathrm{RF}}$ in respect that every coupling bunch mode is involved. Bunch by bunch measurements are of 3 kinds: bunch charge which mearues quantities of electrons filled in the bucket, transverse Betatron oscillation and longitudinal synchronous phase oscillation. The block diagram of the measurement system is represented in Fig.5.

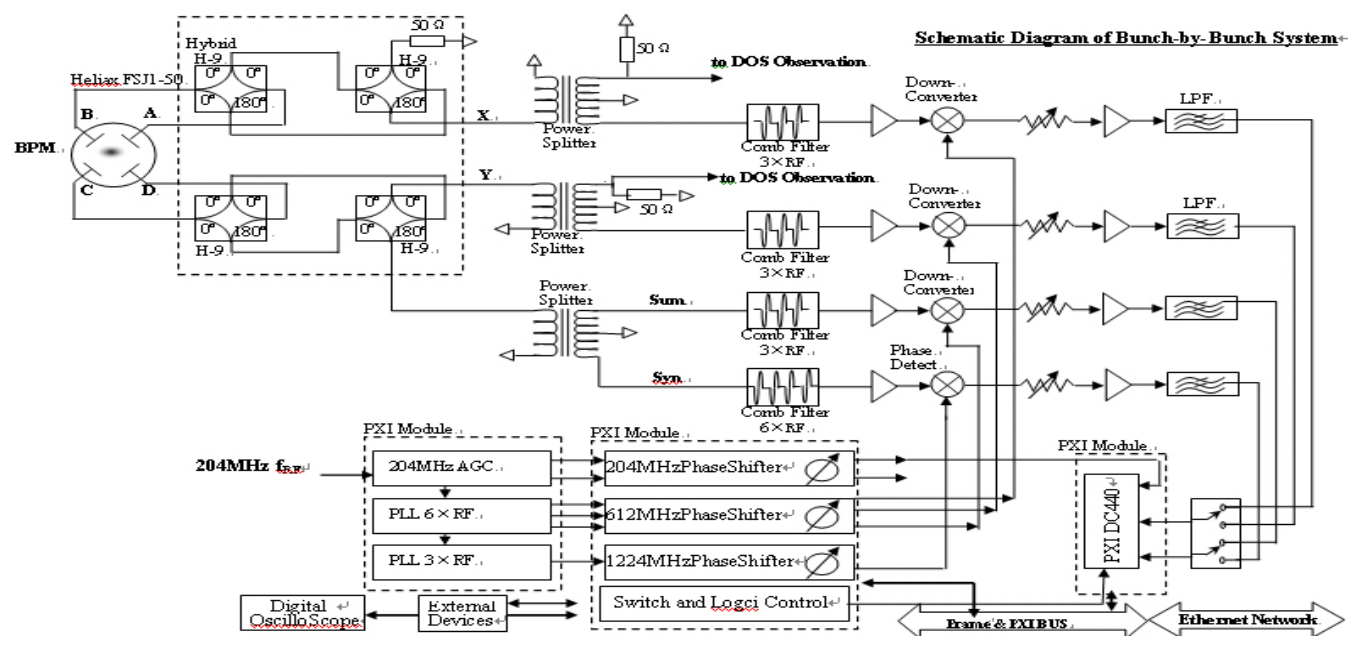

Figure 5: EBlock digarm of bunch by bunch measurement system 
Betatron oscillation and bunch charge measurement is in nature a wideband heterodyne receiving circuit[4]. The function of Magic-T matrix is to produce sum and difference signals which correspond to bunch charge and transverse position respectively. A FIR filter is utilized to narrow the bandwidth of pickup signal and increase signal noise ratio. All frequency components, namely possible modes, distributing in span $\mathrm{f}_{\mathrm{RF}} / 2$ around the center frequency $3 \mathrm{f}_{\mathrm{RF}}$ are extracted and then mixed downward to zero CF. To better suppress the image alias interference, we insert a $100 \mathrm{MHz}$ low pass filter between front end modules and the digitizer.

The synchronous phase oscillation measurement has much in common with the above scheme, except that its signal detection is accomplished by phase detection technique.

The measuremnt system is based on PXI measuremtn and control platform, a fast developing industry standard. Phase look loop frequency multiplier, radio frequency phase and attenuation controller and 12 bit resolution high frequency digitizer are implemend as PXI plug-in modules. The PLL frequency multiplier module is developed with a PE3236 as kernel circuit, producing $3 f_{R F}$ and $6 f_{R F}$ which precisely locks to the ring RF signal. It can also drive RF signal distributed to digitizer and other devices as external clock. To maintain equilibrant between channels, one need to adjust phase, delay, amplitude and switching of signals. This function is implemented in the RF controller module. We employ Acqiris DC440 PXI module as high speed digitizer. It is characterized by 12 bit resolution, as much as 400MSPS, optional external clock, 4M samples storing depth and $\mathrm{PXI} / \mathrm{CPCI}$ compliant. Other function modules are standalone packaged.

\section{RELEVANT APPLICATIONS}

To check the performance of the measurement system we conducted a number of experiments at HLS, including Bunch-by-bunch measurement in stable and quasi-stable conditions, measurement of injection kicker excited oscillation and sbusequent damping, stripline resonantly excited oscillation and damping thereafter, measurement of single bunch by using high switching speed gate, exciting one single bunch with high speed gate.

A couple of new algorithms have been employed, including transient tune estimation (Maximum Entropy Method, Numerical Analysis of Fundamental Frequency, Interpolated FFT, and window correlating algortithms, obtaining better than 0.00005 precision given length of 256), method of time-frequency joined presentation, algorithms to recognize coupled bunch mode[5], computation of phase space tracks and bunch charge estimation.

The results in this paper indicates that there are possible coupling modes: mode number $0,1,14,18,19$ in transverse direction and mode number 0,14 in longitudinal direction respectively. Evolvement of phase space representation and tune shift/spread with time reveals that the dominant beam stability damping mechanism is head-tail damping. Besides Landau damping derived from octuple magnets is also contributing to the stability of stored beam.

Some detailed experiment conditions and relevant results are graphically represented in the following figures.

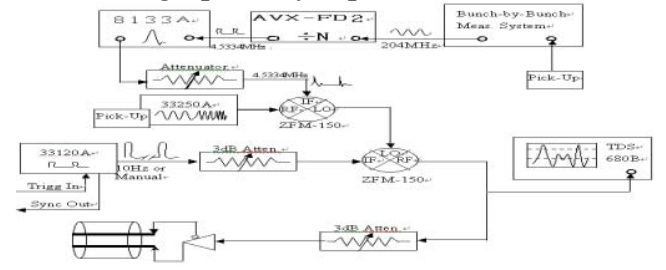

Fig.6 Schematic of single bunch exciting based on fast switch

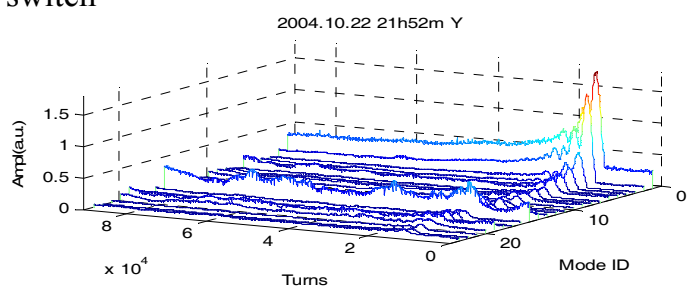

Fig.7 Coupled modes: single bunch exciting in y direction

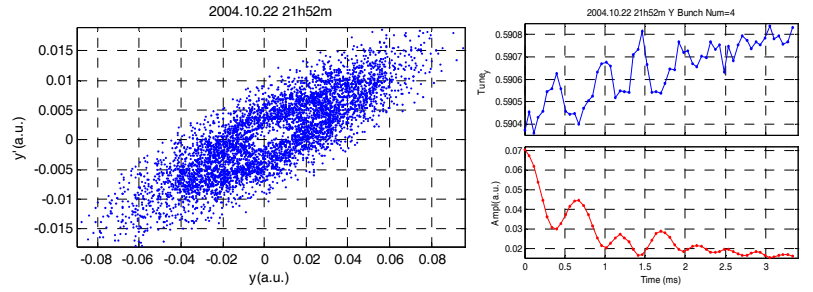

Fig. 8 Evolution of 5000 turns moment in phase space(left) Tune and amplitude vary with turns(right).
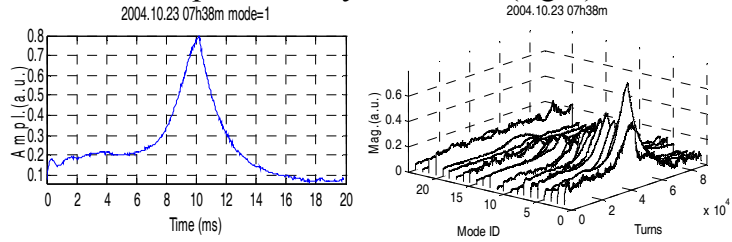

Fig.9 Rising and damping of mode $\mu=1$ (lef)

All possible coupled modes(right)
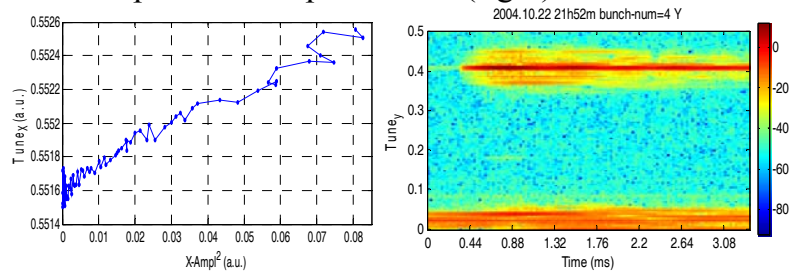

Fig.10 Tune vs. Ampl ${ }^{2}$ (left) and Tune spread(right)

\section{REFERENCES}

[1] M. Serio, DAFNE tech note G-48, 1997.

[2] M. Serio, PAC Conf. Proc., 1999.

[3] J.D. Fox, Joint US-CERN-Japan-Russia school on particle accelerator, 1998.

[4] W. Barry, PAC Conf. Proc., 1995

[5] R. Claus, EPAC Conf. Proc., 1996. 